Research Article

\title{
Sawi Decomposition Method for Volterra Integral Equation with Application
}

\author{
M. Higazy, ${ }^{1,2}$ Sudhanshu Aggarwal $(i),{ }^{3}$ and Taher A. Nofal ${ }^{1}$ \\ ${ }^{1}$ Department of Mathematics and Statistics, College of Science, P.O. Box 11099, Taif University, Taif 21944, Saudi Arabia \\ ${ }^{2}$ Department of Physics and Engineering Mathematics, Faculty of Electronic Engineering, Menoufia University, \\ Menouf 32952, Egypt \\ ${ }^{3}$ Assistant Professor, Department of Mathematics, National P.G. College, Barhalganj, Gorakhpur 273402, Uttar Pradesh, India \\ Correspondence should be addressed to Sudhanshu Aggarwal; sudhanshu30187@gmail.com
}

Received 14 October 2020; Revised 19 November 2020; Accepted 27 November 2020; Published 18 December 2020

Academic Editor: Hijaz Ahmad

Copyright (C) 2020 M. Higazy et al. This is an open access article distributed under the Creative Commons Attribution License, which permits unrestricted use, distribution, and reproduction in any medium, provided the original work is properly cited.

\begin{abstract}
In this paper, authors present a new method "Sawi decomposition method" for determining the primitive of Volterra integral equation (V.I.E.) with application. Sawi decomposition method is the combination of Sawi transformation and decomposition method. Some numerical problems have been considered and solved sequentially for explaining the complete methodology. For the practical application of the Sawi decomposition method, an advance problem of medical science for determining the blood glucose concentration during an intravenous injection has been considered and solved sequentially applying this method. Results of numerical problems depict that the Sawi decomposition method is a very effective decomposition method for determining the primitives of V.I.E.
\end{abstract}

\section{Introduction}

Many complex problems of mathematics, chemistry, biology, astrophysics, and mechanics such as problem of radiative energy transfer, oscillation problems of string and membrane, and problem of momentum representation in quantum mechanics can be expressed in the terms of Volterra integral equation. Aggarwal et al. [1] and Chauhan and Aggarwal [2] used different integral transformations for obtaining the solutions of V.I.E. of second kind. Abdelrahim [3] solved constant coefficient linear differential equations by defining Sawi transformation. Singh and Aggarwal [4] applied Sawi transformation for determining the solutions of biological problems of growth and decay. Aggarwal and Gupta [5] established duality relations between Sawi and other advanced integral transformations. Wang [6] gave the reliable mechanical algorithm for obtaining the numerical solution of famous Volterra integral equation. Maleknejad and Aghazadeh [7] used the Taylor-series expansion method and determined the numerical primitives of second kind V.I.E. with convolution kernel.
Rashidinia and Zarebnia [8] solved the Volterra integral equation by the Sinc-collocation method. Babolian and Davari [9] gave the numerical implementation of the Adomian decomposition method for linear Volterra integral equations of the second kind. Lin et al. [10] used extrapolation of the iterated-collocation method for integral equations of the second kind. Zhang et al. [11] applied Galerkin methods for determining the numerical solution for second-kind Volterra integral equations. Shoukralla et al. [12] used the Barycentric-Maclaurin interpolation method for solving Volterra integral equations of the second kind. Isaacson and Kirby [13] gave the numerical solution of linear Volterra integral equations of the second kind with sharp gradients. The Adomian decomposition method of Volterra integral equation of second kind was given by Abaoud et al. [14].

Aggarwal et al. [15] applied Mahgoub transform for solving linear Volterra integral equations. Aggarwal et al. [16] gave a new application of Shehu transform for handling Volterra integral equations of first kind. Solution of linear volterra integral equations of second kind using mohand 
transform was given by Aggarwal et al. [17]. Aggarwal et al. [18] used Aboodh transform for solving linear Volterra integral equations of first kind. Duality relations of Kamal transform with Laplace, Laplace-Carson, Aboodh, Sumudu, Elzaki, Mohand, and Sawi transforms were given by Aggarwal et al. [19]. Aggarwal and Bhatnagar [20] defined dualities between Laplace transform and some useful integral transforms.

Chauhan et al. [21] gave the dualities between Laplace-Carson transform and some useful integral transforms. Aggarwal and Gupta [22] defined the dualities between Mohand transform and some useful integral transforms. Dualities between Elzaki transform and some useful integral transforms were given by Aggarwal et al. [23]. Chaudhary et al. [24] defined the connections between Aboodh transform and some useful integral transforms. Aggarwal et al. [25] applied Mahgoub transform for solving linear Volterra integral equations of first kind. Application of Elzaki transform for solving linear Volterra integral equations of first kind was given by Aggarwal et al. [26]. Aggarwal and Sharma [27] used Laplace transform for the solution of first kind linear Volterra integral equation. Mishra et al. [28] defined the relationship between Sumudu and some efficient integral transforms. Aggarwal et al. [29] discussed the exact solutions for a class of Wick-type stochastic $(3+1)$-dimensional modified Benjamin-Bona-Mahony equations. Cesarano [30] used generalized special functions in the description of fractional diffusive equations. Dattoli et al. [31] discussed special polynomials and gave some results in fractional calculus. Aggarwal et al. [32] gave the application of Aboodh transform for solving linear Volterra integral equations of first kind. Application of Kamal transform for solving linear volterra integral equations of first kind was given by Aggarwal et al. [33].

The objective of the present paper is to determine the solutions of V.I.Es by applying the Sawi decomposition method on them and determining the solution of the problem of sugar level (blood glucose concentration) of a patient for explaining the applicability of this method in the field of medical science.

\section{Definition of Sawi Transformation}

The Sawi transformation of the function $\omega(t), t \geq 0$ is given by Abdelrahim [3]:

$$
S\{\omega(t)\}=\frac{1}{\sigma^{2}} \int_{0}^{\infty} \omega(t) e^{-(1 / \sigma) t} \mathrm{~d} t=T(\sigma), \quad \sigma>0 .
$$

Remark 1. In the above equation, the function $\left(1 / \sigma^{2}\right) e^{-(1 / \sigma) t}$ is called the kernel of the Sawi transformation.

Remark 2. The Sawi transformation of the function $\omega(t), t \geq 0$ exists if the integral $\left(1 / \sigma^{2}\right) \int_{0}^{\infty} \omega(t) e^{-(1 / \sigma) t} \mathrm{~d} t$ exists.

Remark 3. The integral $\left(1 / \sigma^{2}\right) \int_{0}^{\infty} \omega(t) e^{-(1 / \sigma) t} \mathrm{~d} t$ exists if $\omega(t)$ is a function of exponential order and piecewise continuous in the interval $0 \leq t<\infty$.

\section{Some Important Properties of Sawi Transformation}

Authors present important characteristics of Sawi transformation in this part of the paper.

3.1. Linearity Property of Sawi Transformation. If Sawi transform of functions $\omega_{1}(t)$ and $\omega_{2}(t)$ are $T_{1}(\sigma)$ and $T_{2}(\sigma)$, respectively, then Sawi transform of $\left[l \omega_{1}(t)+m \omega_{2}(t)\right]$ is given by $\left[l T_{1}(\sigma)+m T_{2}(\sigma)\right]$, where $l$ and $m$ are arbitrary constants.

Proof. Using (1), we obtain

$$
\begin{aligned}
S\{\omega(t)\}= & \frac{1}{\sigma^{2}} \int_{0}^{\infty} \omega(t) e^{-(1 / \sigma) t} \mathrm{~d} t \\
\Rightarrow & S\left\{l \omega_{1}(t)+m \omega_{2}(t)\right\} \\
= & \frac{1}{\sigma^{2}} \int_{0}^{\infty}\left[l \omega_{1}(t)+m \omega_{2}(t)\right] e^{-(1 / \sigma) t} \mathrm{~d} t \\
\Rightarrow & S\left\{l \omega_{1}(t)+m \omega_{2}(t)\right\} \\
= & l\left[\frac{1}{\sigma^{2}} \int_{0}^{\infty} \omega_{1}(t) e^{-(1 / \sigma) t} \mathrm{~d} t\right] \\
& +m\left[\frac{1}{\sigma^{2}} \int_{0}^{\infty} \omega_{2}(t) e^{-(1 / \sigma) t} \mathrm{~d} t\right] \\
\Rightarrow & S\left\{l \omega_{1}(t)+m \omega_{2}(t)\right\} \\
= & l S\left\{\omega_{1}(t)\right\}+m S\left\{\omega_{2}(t)\right\} \\
\Rightarrow & S\left\{l \omega_{1}(t)+m \omega_{2}(t)\right\} \\
= & l T_{1}(\sigma)+m T_{2}(\sigma),
\end{aligned}
$$

where $l$ and $m$ are arbitrary constants.

Remark 4. One immediate consequence of the above property is that if $\omega(t)=\sum_{i=1}^{k} a_{i} \omega_{i}(t)$, where $a_{i}$ are arbitrary constants, then $S\{\omega(t)\}=S\left\{\sum_{i=1}^{k} a_{i} \omega_{i}(t)\right\}=\sum_{i=1}^{k} a_{i} S\left\{\omega_{i}(t)\right\}$.

3.2. Scaling Property of Sawi Transformation. If $S\{\omega(t)\}=T(\sigma)$, then $S\{\omega(k t)\}=k T(k \sigma)$.

Proof. Using (1), we obtain

$$
\begin{aligned}
S\{\omega(t)\}= & \frac{1}{\sigma^{2}} \int_{0}^{\infty} \omega(t) e^{-(1 / \sigma) t} \mathrm{~d} t \\
& \Rightarrow S\{\omega(k t)\}=\frac{1}{\sigma^{2}} \int_{0}^{\infty} \omega(k t) e^{-(1 / \sigma) t} \mathrm{~d} t .
\end{aligned}
$$
have 


$$
\begin{aligned}
S\{\omega(k t)\} & =\frac{1}{\sigma^{2}}\left[\frac{1}{k} \int_{0}^{\infty} \omega(p) e^{-(1 / \sigma)(p / k)} \mathrm{d} p\right] \\
& \Rightarrow S\{\omega(k t)\}=\frac{1}{k}\left[\frac{1}{\sigma^{2}} \int_{0}^{\infty} \omega(p) e^{-(1 / \sigma)(p / k)} \mathrm{d} p\right] \\
& =\left[\frac{k}{k^{2} \sigma^{2}} \int_{0}^{\infty} \omega(p) e^{-(1 / k \sigma) p} \mathrm{~d} p\right] \\
& \Rightarrow S\{\omega(k t)\}=k T(k \sigma) .
\end{aligned}
$$

3.3. Translation Property of Sawi Transformation. If $S\{\omega(t)\}=T(\sigma), \quad$ then $\quad S\left\{e^{k t} \omega(t)\right\}=\left(1 /(1-k \sigma)^{2}\right)$ $T(\sigma /(1-k \sigma))$.

Proof. Using (1), we obtain

$$
\begin{aligned}
S\{\omega(t)\} & =\frac{1}{\sigma^{2}} \int_{0}^{\infty} \omega(t) e^{-(1 / \sigma) t} \mathrm{~d} t \\
& \Rightarrow S\left\{e^{k t} \omega(t)\right\}=\frac{1}{\sigma^{2}} \int_{0}^{\infty} e^{k t} \omega(t) e^{-(1 / \sigma) t} \mathrm{~d} t \\
& \Rightarrow S\left\{e^{k t} \omega(t)\right\}=\frac{1}{\sigma^{2}} \int_{0}^{\infty} \omega(t) e^{-((1 / \sigma)-k) t} \mathrm{~d} t \\
& =\frac{1}{\sigma^{2}} \int_{0}^{\infty} \omega(t) e^{-((1-k \sigma) / \sigma) t} \mathrm{~d} t \\
& =\frac{1}{(1-k \sigma)^{2}}\left[\frac{(1-k \sigma)^{2}}{\sigma^{2}} \int_{0}^{\infty} \omega(t) e^{-((1-k \sigma) / \sigma) t} \mathrm{~d} t\right] \\
& =\frac{1}{(1-k \sigma)^{2}} T\left(\frac{\sigma}{1-k \sigma}\right) .
\end{aligned}
$$

\section{Sawi Transformation of Derivatives}

If $S\{\omega(t)\}=T(\sigma)$, then

$$
\begin{gathered}
S\left\{\omega^{\prime}(t)\right\}=\frac{1}{\sigma} T(\sigma)-\frac{1}{\sigma^{2}} \omega(0), \\
S\left\{\omega^{\prime \prime}(t)\right\}=\frac{1}{\sigma^{2}} T(\sigma)-\frac{1}{\sigma^{3}} \omega(0)-\frac{1}{\sigma^{2}} \omega^{\prime}(0), \\
S\left\{\omega^{(\rho)}(t)\right\}=\frac{1}{\sigma^{\rho}} T(\sigma)-\sum_{k=0}^{\rho-1}\left(\frac{1}{\sigma}\right)^{\rho-(k-1)} \omega^{(k)}(0) .
\end{gathered}
$$

Proof

(a) Using (1), we obtain

$$
\begin{aligned}
S\{\omega(t)\}= & \frac{1}{\sigma^{2}} \int_{0}^{\infty} \omega(t) e^{-(1 / \sigma) t} \mathrm{~d} t \\
\Rightarrow & S\left\{\omega^{\prime}(t)\right\}=\frac{1}{\sigma^{2}} \int_{0}^{\infty} \omega^{\prime}(t) e^{-(1 / \sigma) t} \mathrm{~d} t \\
= & \frac{1}{\sigma^{2}}\left[\omega(t) e^{-(1 / \sigma) t}\right]_{0}^{\infty}-\frac{1}{\sigma^{2}} \int_{0}^{\infty}\left\{-\left(\frac{1}{\sigma}\right)\right\} \omega(t) e^{-(1 / \sigma) t} \mathrm{~d} t \\
= & \frac{1}{\sigma^{2}} \lim _{t \rightarrow \infty}\left[\omega(t) e^{-(1 / \sigma) t}\right]-\frac{1}{\sigma^{2}} \omega(0) \\
& +\frac{1}{\sigma^{3}} \int_{0}^{\infty} \omega(t) e^{-(1 / \sigma) t} \mathrm{~d} t=0-\frac{1}{\sigma^{2}} \omega(0)+\frac{1}{\sigma} S\{\omega(t)\} \\
\Rightarrow & S\left\{\omega^{\prime}(t)\right\}=\frac{1}{\sigma} T(\sigma)-\frac{1}{\sigma^{2}} \omega(0) .
\end{aligned}
$$

(b) We have $S\left\{\omega^{\prime}(t)\right\}=(1 / \sigma) T(\sigma)-\left(1 / \sigma^{2}\right) \omega(0)=$ $(1 / \sigma) S\{\omega(t)\}-\left(1 / \sigma^{2}\right) \omega(0)$,

$$
\begin{aligned}
& \Rightarrow S\left\{\omega^{\prime \prime}(t)\right\}=\frac{1}{\sigma} S\left\{\omega^{\prime}(t)\right\}-\frac{1}{\sigma^{2}} \omega^{\prime}(0) \\
& =\frac{1}{\sigma}\left[\frac{1}{\sigma} S\{\omega(t)\}-\frac{1}{\sigma^{2}} \omega(0)\right]-\frac{1}{\sigma^{2}} \omega^{\prime}(0), \\
& \Rightarrow S\left\{\omega^{\prime \prime}(t)\right\}=\frac{1}{\sigma^{2}} S\{\omega(t)\}-\frac{1}{\sigma^{3}} \omega(0)-\frac{1}{\sigma^{2}} \omega^{\prime}(0), \\
& \Rightarrow S\left\{\omega^{\prime \prime}(t)\right\}=\frac{1}{\sigma^{2}} T(\sigma)-\frac{1}{\sigma^{3}} \omega(0)-\frac{1}{\sigma^{2}} \omega^{\prime}(0) .
\end{aligned}
$$

(c) We have $S\left\{\omega^{\prime \prime}(t)\right\}=\left(1 / \sigma^{2}\right) T(\sigma)-\left(1 / \sigma^{3}\right) \omega(0)-$ $\left(1 / \sigma^{2}\right) \omega^{\prime}(0)$,

$=\frac{1}{\sigma^{2}} S\{\omega(t)\}-\frac{1}{\sigma^{3}} \omega(0)-\frac{1}{\sigma^{2}} \omega^{\prime}(0)$

$\Rightarrow S\left\{\omega^{\prime \prime \prime}(t)\right\}=\frac{1}{\sigma^{2}} S\left\{\omega^{\prime}(t)\right\}-\frac{1}{\sigma^{3}} \omega^{\prime}(0)-\frac{1}{\sigma^{2}} \omega^{\prime \prime}(0)$

$=\frac{1}{\sigma^{2}}\left[\frac{1}{\sigma} S\{\omega(t)\}-\frac{1}{\sigma^{2}} \omega(0)\right]-\frac{1}{\sigma^{3}} \omega^{\prime}(0)-\frac{1}{\sigma^{2}} \omega^{\prime \prime}(0)$

$\Rightarrow S\left\{\omega^{\prime \prime \prime}(t)\right\}=\frac{1}{\sigma^{3}} S\{\omega(t)\}-\frac{1}{\sigma^{4}} \omega(0)-\frac{1}{\sigma^{3}} \omega^{\prime}(0)-\frac{1}{\sigma^{2}} \omega^{\prime \prime}(0)$

$=\frac{1}{\sigma^{3}} T(\sigma)-\frac{1}{\sigma^{4}} \omega(0)-\frac{1}{\sigma^{3}} \omega^{\prime}(0)-\frac{1}{\sigma^{2}} \omega^{\prime \prime}(0)$.

In general, we have 


$$
\begin{aligned}
S\left\{\omega^{(\rho)}(t)\right\}= & \frac{1}{\sigma^{\rho}} S\{\omega(t)\}-\frac{1}{\sigma^{\rho+1}} \omega(0) \\
& -\frac{1}{\sigma^{\rho}} \omega^{\prime}(0)-\cdots-\frac{1}{\sigma^{\rho-1}} \omega^{(\rho-1)}(0) \\
\Rightarrow & S\left\{\omega^{(\rho)}(t)\right\}=\frac{1}{\sigma^{\rho}} T(\sigma)-\frac{1}{\sigma^{\rho+1}} \omega(0) \\
& -\frac{1}{\sigma^{\rho}} \omega^{\prime}(0)-\cdots-\frac{1}{\sigma^{\rho-1}} \omega^{(\rho-1)}(0) \\
\Rightarrow & S\left\{\omega^{(\rho)}(t)\right\}=\frac{1}{\sigma^{\rho}} T(\sigma)-\sum_{k=0}^{\rho-1}\left(\frac{1}{\sigma}\right)^{\rho-(k-1)} \omega^{(k)}(0) .
\end{aligned}
$$

Remark 5. The results of this section are very useful and can be used for solving initial value problems.

\section{Faltung Theorem for Sawi Transformation}

Faltung theorem of integral transforms has many applications in the solution of differential equations and integral equations of Faltung form. If $S\left\{\omega_{1}(t)\right\}=T_{1}(\sigma)$ and $S\left\{\omega_{2}(t)\right\}=T_{2}(\sigma)$, then $S\left\{\omega_{1}(t) * \omega_{2}(t)\right\}=S\left\{\omega_{1}(t)\right\} S\left\{\omega_{2}\right.$ $(t)\}=\sigma^{2} T_{1}(\sigma) T_{2}(\sigma)$, where Faltung of $\omega_{1}(t)$ and $\omega_{2}(t)$ is denoted by $\omega_{1}(t) * \omega_{2}(t)$, and it is defined by

$$
\begin{aligned}
\omega_{1}(t) * \omega_{2}(t) & =\int_{0}^{t} \omega_{1}(t-u) \omega_{2}(u) \mathrm{d} u \\
& =\int_{0}^{t} \omega_{1}(u) \omega_{2}(t-u) \mathrm{d} u
\end{aligned}
$$

Proof. Using (1), we obtain

$$
\begin{aligned}
S\{\omega(t)\}= & \frac{1}{\sigma^{2}} \int_{0}^{\infty} \omega(t) e^{-(1 / \sigma) t} \mathrm{~d} t \\
\Rightarrow & S\left\{\omega_{1}(t) * \omega_{2}(t)\right\}=\frac{1}{\sigma^{2}} \int_{0}^{\infty} e^{-(1 / \sigma) t}\left[\omega_{1}(t) * \omega_{2}(t)\right] \mathrm{d} t \\
\Rightarrow & S\left\{\omega_{1}(t) * \omega_{2}(t)\right\}=\frac{1}{\sigma^{2}} \int_{0}^{\infty} e^{-(1 / \sigma) t} \\
& \cdot\left[\int_{0}^{t} \omega_{1}(t-u) \omega_{2}(u) \mathrm{d} u\right] \mathrm{d} t
\end{aligned}
$$

After reversing the order of integration, we obtain

$$
S\left\{\omega_{1}(t) * \omega_{2}(t)\right\}=\frac{1}{\sigma^{2}} \int_{0}^{\infty} \omega_{2}(u)\left[\int_{u}^{\infty} \omega_{1}(t-u) e^{-(1 / \sigma) t} \mathrm{~d} t\right] \mathrm{d} u
$$

Putting $t-u=v$ so that $\mathrm{d} t=\mathrm{d} v$ in the above equation, we have

$$
\begin{aligned}
S\left\{\omega_{1}(t) * \omega_{2}(t)\right\}= & \frac{1}{\sigma^{2}} \int_{0}^{\infty} \omega_{2}(u)\left[\int_{0}^{\infty} \omega_{1}(v) e^{-(1 / \sigma)(v+u)} \mathrm{d} v\right] \mathrm{d} u \\
\Rightarrow & S\left\{\omega_{1}(t) * \omega_{2}(t)\right\} \\
= & \frac{1}{\sigma^{2}} \int_{0}^{\infty} \omega_{2}(u) e^{-(1 / \sigma) u} \\
& \cdot\left[\int_{0}^{\infty} \omega_{1}(v) e^{-(1 / \sigma) v} \mathrm{~d} v\right] \mathrm{d} u \\
\Rightarrow & S\left\{\omega_{1}(t) * \omega_{2}(t)\right\} \\
= & \sigma^{2}\left[\frac{1}{\sigma^{2}} \int_{0}^{\infty} \omega_{2}(u) e^{-(1 / \sigma) u}\right] \\
& \cdot\left[\frac{1}{\sigma^{2}} \int_{0}^{\infty} \omega_{1}(v) e^{-(1 / \sigma) v} \mathrm{~d} v\right] \mathrm{d} u \\
\Rightarrow & S\left\{\omega_{1}(t) * \omega_{2}(t)\right\}=\sigma^{2} T_{1}(\sigma) T_{2}(\sigma) .
\end{aligned}
$$

Remark 6. The above result can be used to obtain a connection between a $k$-fold Faltung of $k$ functions and the product of the transforms of these functions.

Remark 7. In the above definition, the term $\omega_{1}(t-u)$ or $\omega_{2}(t-u)$ is called the influence function.

\section{Inverse Sawi Transformation}

The inverse Sawi transformation of $T(\sigma)$, designated by $S^{-1}\{T(\sigma)\}$, is another function $\omega(t)$ having the property that $S\{\omega(t)\}=T(\sigma)$.

6.1. Linearity Property of Inverse Sawi Transformation. If $S^{-1}\left\{T_{1}(\sigma)\right\}=\omega_{1}(t)$ and $S^{-1}\left\{T_{2}(\sigma)\right\}=\omega_{2}(t)$, then

$$
\begin{aligned}
S^{-1}\left\{l T_{1}(\sigma)+m T_{2}(\sigma)\right\} & =l S^{-1}\left\{T_{1}(\sigma)\right\}+m S^{-1}\left\{T_{2}(\sigma)\right\} \\
& \Rightarrow S^{-1}\left\{l T_{1}(\sigma)+m T_{2}(\sigma)\right\} \\
& =l \omega_{1}(t)+m \omega_{2}(t),
\end{aligned}
$$

where $l$ and $m$ are arbitrary constants.

\section{Sawi Decomposition Method for Volterra Integral Equation}

This section contains Sawi decomposition method for the primitive of linear Faltung-type second kind V.I.E. The general form of second kind linear Faltung-type Volterra integral equation is given by $[2,34-36]$

$$
\omega(t)=g(t)+\mu \int_{0}^{t} K(t-u) \omega(u) \mathrm{d} u,
$$

where 


$$
\begin{aligned}
& \omega(t)=\text { unknown function } \\
& g(t)=\text { known function (perturbation function) } \\
& \mu=\text { non - zero parameter } \\
& K(t-u)=\text { faltung type kernel }
\end{aligned}
$$
have

Operating Sawi transformation on both sides of (16), we

$$
S\{\omega(t)\}=S\{g(t)\}+\mu S\left\{\int_{0}^{t} K(t-u) \omega(u) \mathrm{d} u\right\} .
$$

Applying Faltung theorem of Sawi transformation on (18), we obtain

$$
S\{\omega(t)\}=S\{g(t)\}+\mu \sigma^{2} S\{K(t)\} S\{\omega(t)\} .
$$

Operating inverse Sawi transformation on both sides of (19), we have

$$
\omega(t)=g(t)+\mu S^{-1}\left\{\sigma^{2} S\{K(t)\} S\{\omega(t)\}\right\} .
$$

The Sawi decomposition method assumes the solution $\omega(t)$ of (16) is analytic, so $\omega(t)$ can be expressed in terms of infinite series as

$$
\omega(t)=\sum_{m=0}^{\infty} \omega_{m}(t) .
$$

Using (21) in (20), we obtain

$$
\sum_{m=0}^{\infty} \omega_{m}(t)=g(t)+\mu S^{-1}\left\{\sigma^{2} S\{K(t)\} S\left\{\sum_{m=0}^{\infty} \omega_{m}(t)\right\}\right\} .
$$

In general, the recursive relations are given by

$$
\begin{aligned}
& \omega_{0}(t)=g(t) \\
& \left.\omega_{m+1}(t)=\mu S^{-1}\left\{\sigma^{2} S\{K(t)\} S\left\{\sum_{m=0}^{\infty} \omega_{m}(t)\right\}\right\}, \quad m \geq 0\right\} .
\end{aligned}
$$

Using (23), we can find the values of $\omega_{0}(t)$, $\omega_{1}(t), \omega_{2}(t), \omega_{3}(t), \ldots$ easily. After finding these values, we use (21) for required solution of (16).

Remark 8. The present scheme can be used for determining the primitives of system of linear Faltung-type second kind Volterra integral equations in future.

Sawi and inverse Sawi transformations of frequently used functions are given in Tables 1 and 2, respectively.

Example 1. Consider following linear second kind Faltungtype V.I.E.:

$$
\omega(t)=t+\int_{0}^{t} \omega(u) \mathrm{d} u
$$

Operating Sawi transformation on both sides of (24), we have
TABLE 1: Sawi transformation of frequently used functions $[4,5]$.

\begin{tabular}{lcc}
\hline S.N. & $\omega(t)$ & $S\{\omega(t)\}=T(\sigma)$ \\
\hline 1. & 1 & $(1 / \sigma)$ \\
2. & $t$ & 1 \\
3. & $t^{2}$ & $2 \sigma$ \\
4. & $t^{\rho}, \rho \in N$ & $\rho !(\sigma)^{\rho-1}$ \\
5. & $t^{\rho}, \rho>-1$ & $\Gamma(\rho+1)(\sigma)^{\rho-1}$ \\
6. & $e^{l t}$ & $(1 /(\sigma(1-l \sigma)))$ \\
7. & $\sin l t$ & $\left(l /\left(1+l^{2} \sigma^{2}\right)\right)$ \\
8. & $\cos l t$ & $\left(1 /\left(\sigma\left(1+l^{2} \sigma^{2}\right)\right)\right)$ \\
9. & $\sinh l t$ & $\left(l /\left(1-l^{2} \sigma^{2}\right)\right)$ \\
10. & $\cosh l t$ & $\left(1 /\left(\sigma\left(1-l^{2} \sigma^{2}\right)\right)\right)$ \\
\hline
\end{tabular}

TABLE 2: Inverse Sawi transformation of frequently used functions [4].

\begin{tabular}{lcc}
\hline S.N. & $T(\sigma)$ & $\omega(t)=S^{-1}\{T(\sigma)\}$ \\
\hline 1. & $(1 / \sigma)$ & 1 \\
2. & 1 & $t$ \\
3. & $\sigma$ & $\left(t^{2} / 2 !\right)$ \\
4. & $(\sigma)^{\rho-1}, \rho \in N$ & $\left(t^{\rho} / \rho !\right)$ \\
5. & $(\sigma)^{\rho-1}, \rho>-1$ & $\left(t^{\rho} /(\Gamma(\rho+1))\right)$ \\
6. & $(1 /(\sigma(1-l \sigma)))$ & $e^{l t}$ \\
7. & $\left(1 /\left(1+l^{2} \sigma^{2}\right)\right)$ & $(\sin l t / l)$ \\
8. & $\left(1 /\left(\sigma\left(1+l^{2} \sigma^{2}\right)\right)\right)$ & $\cos l t$ \\
9. & $\left(1 /\left(1-l^{2} \sigma^{2}\right)\right)$ & $(\sinh l t / l)$ \\
10. & $\left(1 /\left(\sigma\left(1-l^{2} \sigma^{2}\right)\right)\right)$ & $\cosh l t$ \\
\hline
\end{tabular}

$$
\begin{aligned}
S\{\omega(t)\} & =S\{t\}+S\left\{\int_{0}^{t} \omega(u) \mathrm{d} u\right\} \\
& \Rightarrow S\{\omega(t)\}=1+S\left\{\int_{0}^{t} \omega(u) \mathrm{d} u\right\} .
\end{aligned}
$$

Applying Faltung theorem of Sawi transformation on (25), we obtain

$$
\begin{aligned}
S\{\omega(t)\} & =1+\sigma^{2} S\{1\} S\{\omega(t)\} \\
& \Rightarrow S\{\omega(t)\}=1+\sigma^{2}\left(\frac{1}{\sigma}\right) S\{\omega(t)\} .
\end{aligned}
$$

Operating inverse Sawi transformation on both sides of (26), we have

$$
\omega(t)=t+S^{-1}\{\sigma S\{\omega(t)\}\} .
$$

The Sawi decomposition method assumes the solution $\omega(t)$ of (24) is analytic so $\omega(t)$ can be expressed in terms of infinite series as

$$
\omega(t)=\sum_{m=0}^{\infty} \omega_{m}(t) .
$$

Using (8) in (27), we obtain

$$
\sum_{m=0}^{\infty} \omega_{m}(t)=t+S^{-1}\left\{\sigma S\left\{\sum_{m=0}^{\infty} \omega_{m}(t)\right\}\right\} .
$$

In general, the recursive relations are given by 


$$
\begin{aligned}
& \omega_{0}(t)=t \\
& \left.\omega_{m+1}(t)=S^{-1}\left\{\sigma S\left\{\sum_{m=0}^{\infty} \omega_{m}(t)\right\}\right\}, \quad m \geq 0\right\}
\end{aligned}
$$

with the help of (30), we obtain

$$
\begin{aligned}
& \omega_{1}(t)=S^{-1}\left\{\sigma S\left\{\omega_{0}(t)\right\}\right\}=S^{-1}\{\sigma S\{t\}\}=S^{-1}\{\sigma \cdot 1\}=S^{-1}\{\sigma\}=\frac{t^{2}}{2} \\
& \omega_{2}(t)=S^{-1}\left\{\sigma S\left\{\omega_{1}(t)\right\}\right\}=S^{-1}\left\{\sigma S\left\{\frac{t^{2}}{2}\right\}\right\}=S^{-1}\{\sigma \cdot \sigma\}=S^{-1}\left\{(\sigma)^{2}\right\}=\frac{t^{3}}{6}, \\
& \omega_{3}(t)=S^{-1}\left\{\sigma S\left\{\omega_{2}(t)\right\}\right\}=S^{-1}\left\{\sigma S\left\{\frac{t^{3}}{6}\right\}\right\}=S^{-1}\left\{\sigma \cdot(\sigma)^{2}\right\}=S^{-1}\left\{(\sigma)^{3}\right\}=\frac{t^{4}}{24}, \\
& \omega_{4}(t)=S^{-1}\left\{\sigma S\left\{\omega_{3}(t)\right\}\right\}=S^{-1}\left\{\sigma S\left\{\frac{t^{4}}{24}\right\}\right\}=S^{-1}\left\{\sigma \cdot(\sigma)^{3}\right\}=S^{-1}\left\{(\sigma)^{4}\right\}=\frac{t^{5}}{120},
\end{aligned}
$$

and so on. Using (28), the series solution of (24) is given by

$$
\begin{aligned}
\omega(t) & =\sum_{m=0}^{\infty} \omega_{m}(t) \\
& \Rightarrow \omega(t)=\left[t+\frac{t^{2}}{2}+\frac{t^{3}}{6}+\frac{t^{4}}{24}+\frac{t^{5}}{120}+\cdots\right]=e^{t}-1 .
\end{aligned}
$$

Example 2. Consider the following linear second kind Faltung-type V.I.E.:

$$
\omega(t)=t-\int_{0}^{t} \omega(u) \mathrm{d} u
$$
have

Operating Sawi transformation on both sides of (33), we

$$
\begin{aligned}
S\{\omega(t)\} & =S\{t\}-S\left\{\int_{0}^{t} \omega(u) \mathrm{d} u\right\}, \\
& \Rightarrow S\{\omega(t)\}=1-S\left\{\int_{0}^{t} \omega(u) \mathrm{d} u\right\} .
\end{aligned}
$$

Applying Faltung theorem of Sawi transformation on (34), we obtain

$$
\begin{aligned}
S\{\omega(t)\} & =1-\sigma^{2} S\{1\} S\{\omega(t)\}, \\
& \Rightarrow S\{\omega(t)\}=1-\sigma^{2}\left(\frac{1}{\sigma}\right) S\{\omega(t)\} .
\end{aligned}
$$

Operating inverse Sawi transformation on both sides of (35), we have

$$
\omega(t)=t-S^{-1}\{\sigma S\{\omega(t)\}\}
$$

The Sawi decomposition method assumes the solution $\omega(t)$ of (33) is analytic, so $\omega(t)$ can be expressed in terms of infinite series as

$$
\omega(t)=\sum_{m=0}^{\infty} \omega_{m}(t)
$$

Using (37) in (36), we obtain

$$
\sum_{m=0}^{\infty} \omega_{m}(t)=t-S^{-1}\left\{\sigma S\left\{\sum_{m=0}^{\infty} \omega_{m}(t)\right\}\right\} .
$$

In general, the recursive relations are given by

$$
\begin{aligned}
& \omega_{0}(t)=t \\
& \left.\omega_{m+1}(t)=-S^{-1}\left\{\sigma S\left\{\sum_{m=0}^{\infty} \omega_{m}(t)\right\}\right\}, m \geq 0\right\} .
\end{aligned}
$$


With the help of (39), we obtain

$$
\begin{aligned}
& \omega_{1}(t)=-S^{-1}\left\{\sigma S\left\{\omega_{0}(t)\right\}\right\}=-S^{-1}\{\sigma S\{t\}\}=-S^{-1}\{\sigma \cdot 1\}=-S^{-1}\{\sigma\}=-\frac{t^{2}}{2} \\
& \omega_{2}(t)=-S^{-1}\left\{\sigma S\left\{\omega_{1}(t)\right\}\right\}=-S^{-1}\left\{\sigma S\left\{-\frac{t^{2}}{2}\right\}\right\}=S^{-1}\{\sigma \cdot \sigma\}=S^{-1}\left\{(\sigma)^{2}\right\}=\frac{t^{3}}{6} \\
& \omega_{3}(t)=-S^{-1}\left\{\sigma S\left\{\omega_{2}(t)\right\}\right\}=-S^{-1}\left\{\sigma S\left\{\frac{t^{3}}{6}\right\}\right\}=-S^{-1}\left\{\sigma \cdot(\sigma)^{2}\right\}=-S^{-1}\left\{(\sigma)^{3}\right\}=-\frac{t^{4}}{24} \\
& \omega_{4}(t)=-S^{-1}\left\{\sigma S\left\{\omega_{3}(t)\right\}\right\}=-S^{-1}\left\{\sigma S\left\{-\frac{t^{4}}{24}\right\}\right\}=S^{-1}\left\{\sigma \cdot(\sigma)^{3}\right\}=S^{-1}\left\{(\sigma)^{4}\right\}=\frac{t^{5}}{120}
\end{aligned}
$$

and so on. Using (37), the series solution of (33) is given by

$$
\omega(t)=\sum_{m=0}^{\infty} \omega_{m}(t)=t-\frac{t^{2}}{2}+\frac{t^{3}}{6}-\frac{t^{4}}{24}+\frac{t^{5}}{120}-\cdots=1-e^{-t} .
$$

\section{Application}

This part of the paper contains an application from the field of medical science during an intravenous injection (continuous) for determining blood glucose concentration $C(t)$ of a patient at any particular time $t$. This concentration $C(t)$ is determined by the following linear Volterra integral equation:

$$
C(t)=C_{i}+\left(\frac{\alpha}{V}\right) t-k \int_{0}^{t} C(x) \mathrm{d} x,
$$

where

$$
\left.\begin{array}{l}
k: \text { constant velocity of elimination } \\
\alpha: \text { the rate of infusion } \\
V: \text { volume in which glucose is distributed } \\
C_{i}: \text { initial concentration of glucose in the blood }
\end{array}\right\}
$$
have

Operating Sawi transformation on both sides of (42), we

$$
\begin{aligned}
S\{C(t)\} & =C_{i} S\{1\}+\left(\frac{\alpha}{V}\right) S\{t\}-k S\left\{\int_{0}^{t} C(x) \mathrm{d} x\right\}, \\
& \Rightarrow S\{C(t)\}=C_{i}\left(\frac{1}{\sigma}\right)+\left(\frac{\alpha}{V}\right) \cdot 1-k S\left\{\int_{0}^{t} C(x) \mathrm{d} x\right\} .
\end{aligned}
$$

Applying Faltung theorem of Sawi transformation on (44), we obtain

$$
\begin{aligned}
S\{C(t)\} & =C_{i}\left(\frac{1}{\sigma}\right)+\left(\frac{\alpha}{V}\right)-k \sigma^{2} S\{1\} S\{C(t)\}, \\
& \Rightarrow S\{C(t)\}=C_{i}\left(\frac{1}{\sigma}\right)+\left(\frac{\alpha}{V}\right)-k \sigma^{2}\left(\frac{1}{\sigma}\right) S\{C(t)\} .
\end{aligned}
$$

Operating inverse Sawi transformation on both sides of (45), we have

$$
C(t)=C_{i}+\left(\frac{\alpha}{V}\right) t-k S^{-1}\{\sigma S\{C(t)\}\} .
$$

The Sawi decomposition method assumes the solution $C(t)$ of (42) is analytic, so it can be represent in power series (Taylor's series) as

$$
C(t)=\sum_{m=0}^{\infty} C_{m}(t)
$$

Using (47) in (46), we obtain

$$
\sum_{m=0}^{\infty} C_{m}(t)=C_{i}+\left(\frac{\alpha}{V}\right) t-k S^{-1}\left\{\sigma S\left\{\sum_{m=0}^{\infty} C_{m}(t)\right\}\right\} .
$$

In general, the recursive relations are given by

$$
\left.\begin{array}{l}
C_{0}(t)=C_{i}+\left(\frac{\alpha}{V}\right) t \\
C_{m+1}(t)=-k S^{-1}\left\{\sigma S\left\{\sum_{m=0}^{\infty} C_{m}(t)\right\}\right\}, m \geq 0
\end{array}\right\} .
$$

Using (49), we obtain 


$$
\begin{aligned}
& C_{1}(t)=-k S^{-1}\left\{\sigma S\left\{C_{0}(t)\right\}\right\}=-k S^{-1}\left\{\sigma S\left\{C_{i}+\left(\frac{\alpha}{V}\right) t\right\}\right\}=-k S^{-1}\left\{\sigma\left[S\left\{C_{i}\right\}+S\left\{\left(\frac{\alpha}{V}\right) t\right\}\right]\right\} \\
& =-C_{i} k S^{-1}\{\sigma S\{1\}\}-\left(\frac{\alpha}{V}\right) k S^{-1}\{\sigma S\{t\}\}=-C_{i} k S^{-1}\left\{\sigma \cdot\left(\frac{1}{\sigma}\right)\right\}-k\left(\frac{\alpha}{V}\right) S^{-1}\{\sigma \cdot 1\} \\
& =-C_{i} k S^{-1}\{1\}-k\left(\frac{\alpha}{V}\right) S^{-1}\{\sigma\}=-k\left[C_{i} t+\left(\frac{\alpha}{V}\right) \frac{t^{2}}{2 !}\right], \\
& C_{2}(t)=-k S^{-1}\left\{\sigma S\left\{C_{1}(t)\right\}\right\}=-k S^{-1}\left\{\sigma S\left\{-k\left[C_{i} t+\left(\frac{\alpha}{V}\right) \frac{t^{2}}{2 !}\right]\right\}\right\} \\
& =k^{2} S^{-1}\left\{\sigma\left[S\left\{C_{i} t\right\}+S\left\{\left(\frac{\alpha}{V}\right) \frac{t^{2}}{2 !}\right\}\right]\right\} \\
& =k^{2}\left[C_{i} S^{-1}\{\sigma S\{t\}\}+\left(\frac{\alpha}{V}\right) S^{-1}\left\{\sigma S\left\{\frac{t^{2}}{2 !}\right\}\right\}\right] \\
& =k^{2}\left[C_{i} S^{-1}\{\sigma \cdot 1\}+\left(\frac{\alpha}{V}\right) S^{-1}\{\sigma \cdot \sigma\}\right] \\
& =k^{2}\left[C_{i} S^{-1}\{\sigma\}+\left(\frac{\alpha}{V}\right) S^{-1}\left\{(\sigma)^{2}\right\}\right]=k^{2}\left[C_{i} \frac{t^{2}}{2 !}+\left(\frac{\alpha}{V}\right) \frac{t^{3}}{3 !}\right], \\
& C_{3}(t)=-k S^{-1}\left\{\sigma S\left\{C_{2}(t)\right\}\right\}=-k S^{-1}\left\{\sigma S\left\{k^{2}\left[C_{i} \frac{t^{2}}{2 !}+\left(\frac{\alpha}{V}\right) \frac{t^{3}}{3 !}\right]\right\}\right\} \\
& =-k^{3} S^{-1}\left\{\sigma\left[S\left\{C_{i} \frac{t^{2}}{2 !}\right\}+S\left\{\left(\frac{\alpha}{V}\right) \frac{t^{3}}{3 !}\right\}\right]\right\}=-k^{3}\left[C_{i} S^{-1}\left\{\sigma S\left\{\frac{t^{2}}{2 !}\right\}\right\}+\left(\frac{\alpha}{V}\right) S^{-1}\left\{\sigma S\left\{\frac{t^{3}}{3 !}\right\}\right\}\right] \\
& =-k^{3}\left[C_{i} S^{-1}\{\sigma \cdot \sigma\}+\left(\frac{\alpha}{V}\right) S^{-1}\left\{\sigma \cdot(\sigma)^{2}\right\}\right]=-k^{3}\left[C_{i} S^{-1}\left\{(\sigma)^{2}\right\}+\left(\frac{\alpha}{V}\right) S^{-1}\left\{(\sigma)^{3}\right\}\right] \\
& =-k^{3}\left[C_{i} \frac{t^{3}}{3 !}+\left(\frac{\alpha}{V}\right) \frac{t^{4}}{4 !}\right] \\
& C_{4}(t)=-k S^{-1}\left\{\sigma S\left\{C_{3}(t)\right\}\right\}=-k S^{-1}\left\{\sigma S\left\{-k^{3}\left[C_{i} \frac{t^{3}}{3 !}+\left(\frac{\alpha}{V}\right) \frac{t^{4}}{4 !}\right]\right\}\right\} \\
& =k^{4} S^{-1}\left\{\sigma\left[S\left\{C_{i} \frac{t^{3}}{3 !}\right\}+S\left\{\left(\frac{\alpha}{V}\right) \frac{t^{4}}{4 !}\right\}\right]\right\}=k^{4}\left[C_{i} S^{-1}\left\{\sigma S\left\{\frac{t^{3}}{3 !}\right\}\right\}+\left(\frac{\alpha}{V}\right) S^{-1}\left\{\sigma S\left\{\frac{t^{4}}{4 !}\right\}\right\}\right] \\
& =k^{4}\left[C_{i} S^{-1}\left\{\sigma \cdot(\sigma)^{2}\right\}+\left(\frac{\alpha}{V}\right) S^{-1}\left\{\sigma \cdot(\sigma)^{3}\right\}\right]=k^{4}\left[C_{i} S^{-1}\left\{(\sigma)^{3}\right\}+\left(\frac{\alpha}{V}\right) S^{-1}\left\{(\sigma)^{4}\right\}\right] \\
& =k^{4}\left[C_{i} \frac{t^{4}}{4 !}+\left(\frac{\alpha}{V}\right) \frac{t^{5}}{5 !}\right]
\end{aligned}
$$


and so on. Using (47), the series solution of (42) is given by

$$
\begin{aligned}
C(t) & =\sum_{m=0}^{\infty} C_{m}(t) \\
& =\left[C_{i}+\left(\frac{\alpha}{V}\right)\right] t-k\left[C_{i} t+\left(\frac{\alpha}{V}\right) \frac{t^{2}}{2 !}\right]+k^{2}\left[C_{i} \frac{t^{2}}{2 !}+\left(\frac{\alpha}{V}\right) \frac{t^{3}}{3 !}\right]-k^{3}\left[C_{i} \frac{t^{3}}{3 !}+\left(\frac{\alpha}{V}\right) \frac{t^{4}}{4 !}\right]+k^{4}\left[C_{i} \frac{t^{4}}{4 !}+\left(\frac{\alpha}{V}\right) \frac{t^{5}}{5 !}\right]+\cdots \\
& =C_{i}\left[1-k t+\frac{k^{2}}{2 !} t^{2}-\frac{k^{3}}{3 !} t^{3}+\frac{k^{4}}{4 !} t^{4}-\cdots\right]+\left(\frac{\alpha}{V}\right)\left[t-\frac{k}{2 !} t^{2}+\frac{k^{2}}{3 !} t^{3}-\frac{k^{3}}{4 !} t^{4}+\frac{k^{4}}{5 !} t^{5}-\cdots\right] \\
& =C_{i} e^{-k t}+\left(\frac{\alpha}{V}\right)\left(\frac{k}{k}\right)\left[t-\frac{k}{2} t^{2}+\frac{k^{2}}{6} t^{3}-\frac{k^{3}}{24} t^{4}+\frac{k^{4}}{120} t^{5}-\cdots\right] \\
& =C_{i} e^{-k t}+\left(\frac{\alpha}{V k}\right)\left[k t-\frac{k^{2}}{2} t^{2}+\frac{k^{3}}{6} t^{3}-\frac{k^{4}}{24} t^{4}+\frac{k^{5}}{120} t^{5}-\cdots\right] \\
& =C_{i} e^{-k t}+\left(\frac{\alpha}{V k}\right)\left[1-1+k t-\frac{k^{2}}{2} t^{2}+\frac{k^{3}}{6} t^{3}-\frac{k^{4}}{24} t^{4}+\frac{k^{5}}{120} t^{5}-\cdots\right] \\
C(t) & =C_{i} e^{-k t}+\left(\frac{\alpha}{V k}\right)\left[1-e^{-k t}\right] \cdot \\
& =C_{i} e^{-k t}+\left(\frac{\alpha}{V k}\right)\left[1-\left\{1-k t+\frac{k^{2}}{2} t^{2}-\frac{k^{3}}{6} t^{3}+\frac{k^{4}}{24} t^{4}-\frac{k^{5}}{120} t^{5}+\cdots\right\}\right]
\end{aligned}
$$

The values of blood glucose concentration $C(t)$ are obtained for different values of initial concentration of glucose $C_{i}$, volume in which glucose is distributed $V$, the rate of infusion $\alpha$, constant velocity of elimination $k$, and time $t$. All these results are presented in Tables 3-6.

The normal range of blood glucose mentioned by American Diabetes Association [37] is 79 to $110 \mathrm{mg} / \mathrm{dL}$.

From Table 3, it can be concluded that, as time $t$ increases from 0 to $90 \mathrm{~min}$, blood glucose concentration $C(t)$ decreases for all the five combinations, namely, $\{V=45 \mathrm{dL}, \alpha=$ (280 mg/min), $k=0.058 \mathrm{~min}^{-1}, C_{i}=320 \mathrm{mg} / \mathrm{dL} V=45 \mathrm{dL}$, $\alpha=(280 \mathrm{mg} / \mathrm{min}), \quad k=0.058 \mathrm{~min}^{-1}, C_{i}=322 \mathrm{mg} / \mathrm{dL} \quad V=$ $45 \mathrm{dL}, \alpha=(280 \mathrm{mg} / \mathrm{min}), k=0.058 \mathrm{~min}^{-1}, C_{i}=324 \mathrm{mg} / \mathrm{dL}$ $V=45 \mathrm{dL}, \quad \alpha=(280 \mathrm{mg} / \mathrm{min}), k=0.058 \mathrm{~min}^{-1}, \quad C_{i}=$ $326 \mathrm{mg} / \mathrm{dL} V=45 \mathrm{dL}, \alpha=(280 \mathrm{mg} / \mathrm{min}), k=0.058 \mathrm{~min}^{-1}$, $\left.C_{i}=328 \mathrm{mg} / \mathrm{dL}\right\}$. From this table, it is also clear that the normal blood glucose concentration is achieved in $90 \mathrm{~min}$ for all five combinations. The graph plotted in Figure 1 supports the results of Table 3 .

From Table 4, it can be concluded that, as time $t$ increases from 0 to $90 \mathrm{~min}$, blood glucose concentration $C(t)$ decreases for all the five combinations, namely, $\left\{C_{i}=\right.$ $325 \mathrm{mg} / \mathrm{dL}, \alpha=280 \mathrm{mg} / \mathrm{min}, \quad k=0.058 \mathrm{~min}^{-1}, V=45 \mathrm{dL}$ $C_{i}=325 \mathrm{mg} / \mathrm{dL}, \quad \alpha=280 \mathrm{mg} / \mathrm{min}, k=0.058 \mathrm{~min}^{-1}, V=$ $46 \mathrm{dLC}_{i}=325 \mathrm{mg} / \mathrm{dL}, \alpha=280 \mathrm{mg} / \mathrm{min}, \quad k=0.058 \mathrm{~min}^{-1}$, $V=47 \mathrm{dL} \quad C_{i}=325 \mathrm{mg} / \mathrm{dL}, \alpha=280 \mathrm{mg} / \mathrm{min}, k=$ $0.058 \mathrm{~min}^{-1}, \quad V=48 \mathrm{dLC} C_{i}=325 \mathrm{mg} / \mathrm{dL}, \alpha=280 \mathrm{mg} / \mathrm{min}$, $\left.k=0.058 \mathrm{~min}^{-1}, V=49 \mathrm{dL}\right\}$. From this table, it is also clear that the time required for achieving normal blood glucose concentration reduces as volume in which glucose is distributed $V$ increases. The results of Table 4 are supported by the graph, which is plotted in Figure 2.

Table 5 shows that, as time $t$ increases from 0 to $90 \mathrm{~min}$, blood glucose concentration $C(t)$ decreases for all the five combinations, namely, $\left\{V=45 \mathrm{dL}, C_{i}=320 \mathrm{mg} / \mathrm{dL}, k=\right.$ $0.058 \mathrm{~min}^{-1}, \quad \alpha=280 \mathrm{mg} / \mathrm{min} V=45 \mathrm{dL}, C_{i}=320 \mathrm{mg} / \mathrm{dL}$, $k=0.058 \mathrm{~min}^{-1}, \alpha=281 \mathrm{mg} / \mathrm{min} V=45 \mathrm{dL}, \quad C_{i}=320 \mathrm{mg} /$ $\mathrm{dL}, k=0.058 \mathrm{~min}^{-1}, \quad \alpha=282 \mathrm{mg} / \mathrm{min} V=45 \mathrm{dL}, C_{i}=$ $320 \mathrm{mg} / \mathrm{dL}, k=0.058 \mathrm{~min}^{-1}, \quad \alpha=283 \mathrm{mg} / \mathrm{min} V=45 \mathrm{dL}$, $\left.C_{i}=320 \mathrm{mg} / \mathrm{dL}, k=0.058 \mathrm{~min}^{-1}, \alpha=284 \mathrm{mg} / \mathrm{min}\right\}$. From this table, it is also clear that the time required for achieving normal blood glucose concentration increases as the rate of infusion $\alpha$ increases. The graph of Figure 3 supports the results of Table 5 .

Table 6 shows that, as time $t$ increases from 0 to $90 \mathrm{~min}$, blood glucose concentration $C(t)$ decreases for all the five combinations, namely, $\left\{V=45 \mathrm{dL}, \alpha=280 \mathrm{mg} / \mathrm{min}, C_{i}=\right.$ $320 \mathrm{mg} / \mathrm{dL}, k=0.058 \mathrm{~min}^{-1} V=45 \mathrm{dL}, \quad \alpha=280 \mathrm{mg} / \mathrm{min}$, $C_{i}=320 \mathrm{mg} / \mathrm{dL}, k=0.059 \mathrm{~min}^{-1} V=45 \mathrm{dL}, \quad \alpha=280$ $\mathrm{mg} / \mathrm{min}, C_{i}=320 \mathrm{mg} / \mathrm{dL}, \quad k=0.060 \mathrm{~min}^{-1} V=45 \mathrm{dL}, \alpha=$ $280 \mathrm{mg} / \mathrm{min}, C_{i}=320 \mathrm{mg} / \mathrm{dL}, \quad k=0.061 \mathrm{~min}^{-1} V=45 \mathrm{dL}$, $\left.\alpha=280 \mathrm{mg} / \mathrm{min}, C_{i}=320 \mathrm{mg} / \mathrm{dL}, k=0.062 \mathrm{~min}^{-1}\right\}$. From this table, it is also clear that the time required for achieving normal blood glucose concentration decreases as the constant velocity of elimination $k$ increases. The results of 
TABLE 3: Blood glucose concentration $C(t)$ for different values of initial concentration of glucose $C_{i}$ and time $t$ with $V=45 \mathrm{dL}, \alpha=280 \mathrm{mg} / \mathrm{min}$, and $k=0.058 \mathrm{~min}^{-1}$, see Figure 1 .

\begin{tabular}{|c|c|c|c|c|c|}
\hline \multirow{2}{*}{$t(\min )$} & \multicolumn{5}{|c|}{$V=45 \mathrm{dL}, \alpha=280 \mathrm{mg} / \mathrm{min}, k=0.058 \mathrm{~min}^{-1}$} \\
\hline & $C_{i}=320 \mathrm{mg} / \mathrm{dL}$ & $C_{i}=322 \mathrm{mg} / \mathrm{dL}$ & $C_{i}=324 \mathrm{mg} / \mathrm{dL}$ & $C_{i}=326 \mathrm{mg} / \mathrm{dL}$ & $C_{i}=328 \mathrm{mg} / \mathrm{dL}$ \\
\hline 0 & 320 & 322 & 324 & 326 & 328 \\
\hline 15 & 196.40 & 197.24 & 198.08 & 198.91 & 199.75 \\
\hline 30 & 144.62 & 144.97 & 145.32 & 145.67 & 146.02 \\
\hline 45 & 122.92 & 123.07 & 123.22 & 123.36 & 123.51 \\
\hline 60 & 113.83 & 113.89 & 113.96 & 114.02 & 114.08 \\
\hline 75 & 110.03 & 110.05 & 110.08 & 110.10 & 110.13 \\
\hline 90 & 108.43 & 108.44 & 108.45 & 108.46 & 108.47 \\
\hline
\end{tabular}

TABle 4: Blood glucose concentration $C(t)$ for different values of volume in which glucose is distributed $V$ and time $t$ with $C_{i}=325 \mathrm{mg} / \mathrm{dL}, \alpha=280 \mathrm{mg} / \mathrm{min}$, and $k=0.058 \mathrm{~min}^{-1}$, see Figure 2.

\begin{tabular}{|c|c|c|c|c|c|}
\hline \multirow{2}{*}{$t(\min )$} & \multicolumn{5}{|c|}{$C_{i}=325 \mathrm{mg} / \mathrm{dL}, \alpha=280 \mathrm{mg} / \mathrm{min}, k=0.058 \mathrm{~min}^{-1}$} \\
\hline & $V=45 \mathrm{dL}$ & $V=46 \mathrm{dL}$ & $V=47 \mathrm{dL}$ & $V=48 \mathrm{dL}$ & $V=49 \mathrm{dL}$ \\
\hline 0 & 325 & 325 & 325 & 325 & 325 \\
\hline 15 & 198.49 & 197.14 & 195.84 & 194.60 & 193.41 \\
\hline 30 & 145.49 & 143.57 & 141.73 & 139.97 & 138.27 \\
\hline 45 & 123.29 & 121.13 & 119.06 & 117.08 & 115.18 \\
\hline 60 & 113.99 & 111.73 & 109.56 & 107.49 & 105.50 \\
\hline 75 & 110.09 & 107.79 & 105.58 & 103.47 & 101.45 \\
\hline 90 & 108.46 & 106.14 & 103.92 & 101.79 & 99.75 \\
\hline
\end{tabular}

TABle 5: Blood glucose concentration $C(t)$ for different values of the rate of infusion $\alpha$ and time $t$ with $V=45 \mathrm{dL}, C_{i}=320 \mathrm{mg} / \mathrm{dL}$, and $k=0.058 \mathrm{~min}^{-1}$, see Figure 3 .

\begin{tabular}{|c|c|c|c|c|c|}
\hline \multirow{2}{*}{$t(\min )$} & \multicolumn{5}{|c|}{$V=45 \mathrm{dL}, C_{i}=320 \mathrm{mg} / \mathrm{dL}, k=0.058 \mathrm{~min}^{-1}$} \\
\hline & $\alpha=280 \mathrm{mg} / \mathrm{min}$ & $\alpha=281 \mathrm{mg} / \mathrm{min}$ & $\alpha=282 \mathrm{mg} / \mathrm{min}$ & $\alpha=283 \mathrm{mg} / \mathrm{min}$ & $\alpha=284 \mathrm{mg} / \mathrm{min}$ \\
\hline 0 & 320 & 320 & 320 & 320 & 320 \\
\hline 15 & 196.40 & 196.62 & 196.84 & 197.07 & 197.29 \\
\hline 30 & 144.62 & 144.93 & 145.25 & 145.56 & 145.88 \\
\hline 45 & 122.92 & 123.28 & 123.63 & 123.99 & 124.34 \\
\hline 60 & 113.83 & 114.20 & 114.58 & 114.95 & 115.32 \\
\hline 75 & 110.03 & 110.40 & 110.78 & 111.16 & 111.54 \\
\hline 90 & 108.43 & 108.81 & 109.19 & 109.57 & 109.95 \\
\hline
\end{tabular}

TABLE 6: Blood glucose concentration $C(t)$ for different values of constant velocity of elimination $k$ and time $t$ with $V=45 \mathrm{dL}, \alpha=280 \mathrm{mg} / \mathrm{min}$, and $C_{i}=320 \mathrm{mg} / \mathrm{dL}$, see Figure 4 .

\begin{tabular}{|c|c|c|c|c|c|}
\hline \multirow{2}{*}{$t(\min )$} & \multicolumn{5}{|c|}{$V=45 \mathrm{dL}, \alpha=280 \mathrm{mg} / \mathrm{min}, C_{i}=320 \mathrm{mg} / \mathrm{dL}$} \\
\hline & $k=0.058 \mathrm{~min}^{-1}$ & $k=0.059 \mathrm{~min}^{-1}$ & $k=0.060 \mathrm{~min}^{-1}$ & $k=0.061 \mathrm{~min}^{-1}$ & $k=0.062 \mathrm{~min}^{-1}$ \\
\hline 0 & 320 & 320 & 320 & 320 & 320 \\
\hline 15 & 196.40 & 194 & 191.64 & 189.31 & 187.02 \\
\hline 30 & 144.62 & 142 & 139.46 & 136.97 & 134.55 \\
\hline 45 & 122.92 & 120.54 & 118.24 & 116.01 & 113.85 \\
\hline 60 & 113.83 & 111.69 & 109.61 & 107.61 & 105.68 \\
\hline 75 & 110.03 & 108.03 & 106.11 & 104.25 & 102.46 \\
\hline 90 & 108.43 & 106.52 & 104.68 & 102.90 & 101.19 \\
\hline
\end{tabular}




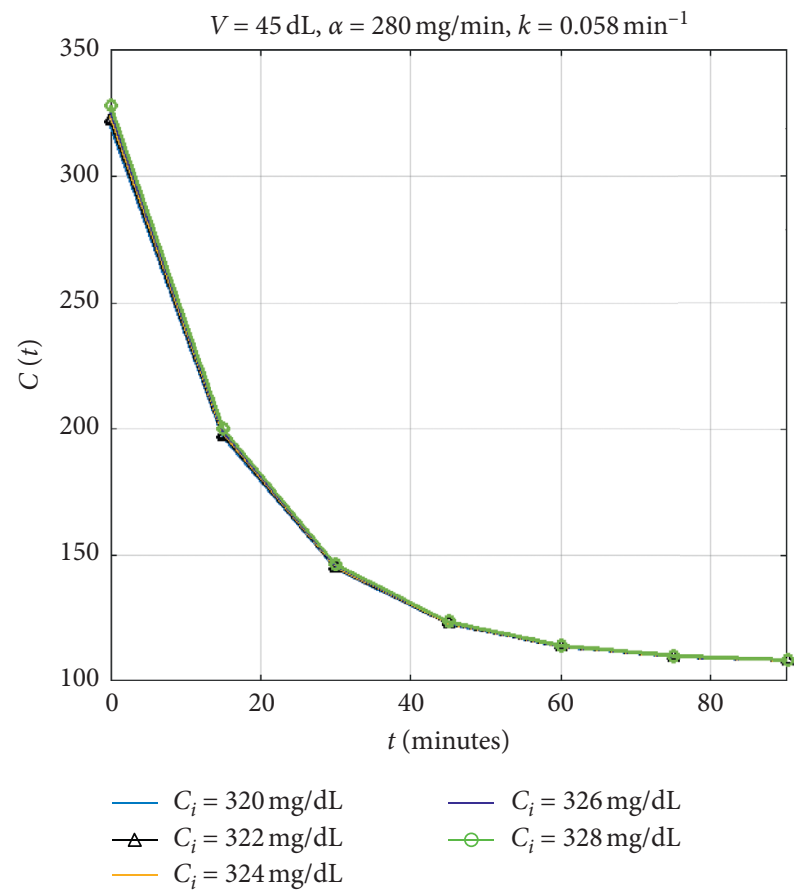

Figure 1: Blood glucose concentration $C(t)$ for different values of initial concentration of glucose $C_{i}$ and time $t$ with. $V=45 \mathrm{dL}, \alpha=280 \mathrm{mg} / \mathrm{min}$, and $k=0.058 \mathrm{~min}^{-1}$.

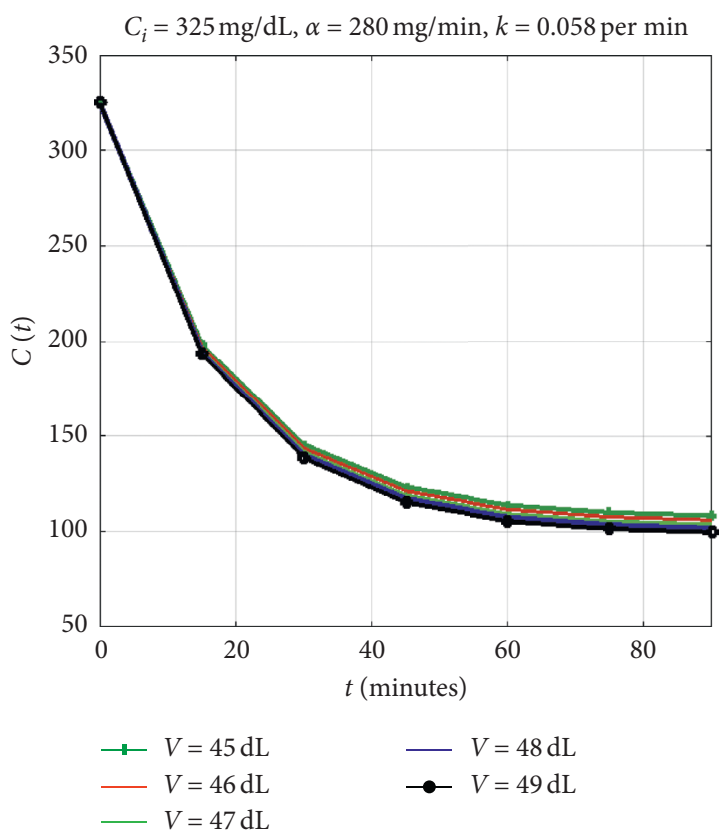

Figure 2: Blood glucose concentration $C(t)$ for different values of volume in which glucose is distributed $V$ and time $t$ with. $C_{i}=325 \mathrm{mg} / \mathrm{dL}, \alpha=280 \mathrm{mg} / \mathrm{min}$, and $k=0.058 \mathrm{~min}^{-1}$.

Table 6 are supported by the graph which is plotted in Figure 4.

\section{Conclusions}

In the present paper, authors fruitfully discussed the Sawi decomposition method for V.I.E. and complete

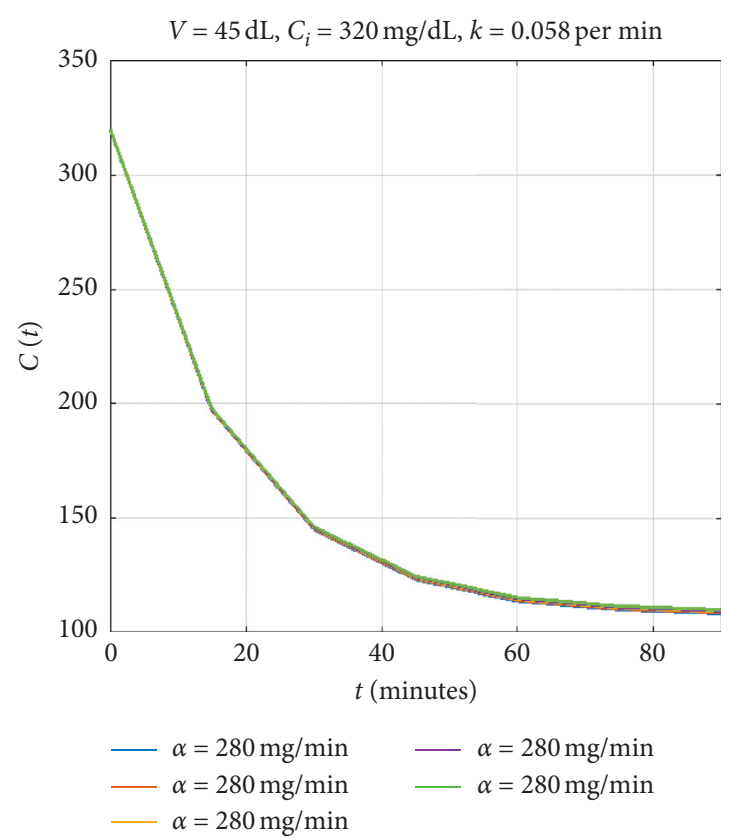

FIgURE 3: Blood glucose concentration $C(t)$ for different values of the rate of infusion $\alpha$ and time $t$ with. $V=45 \mathrm{dL}, C_{i}=320 \mathrm{mg} / \mathrm{dL}$, and $k=0.058 \mathrm{~min}^{-1}$.

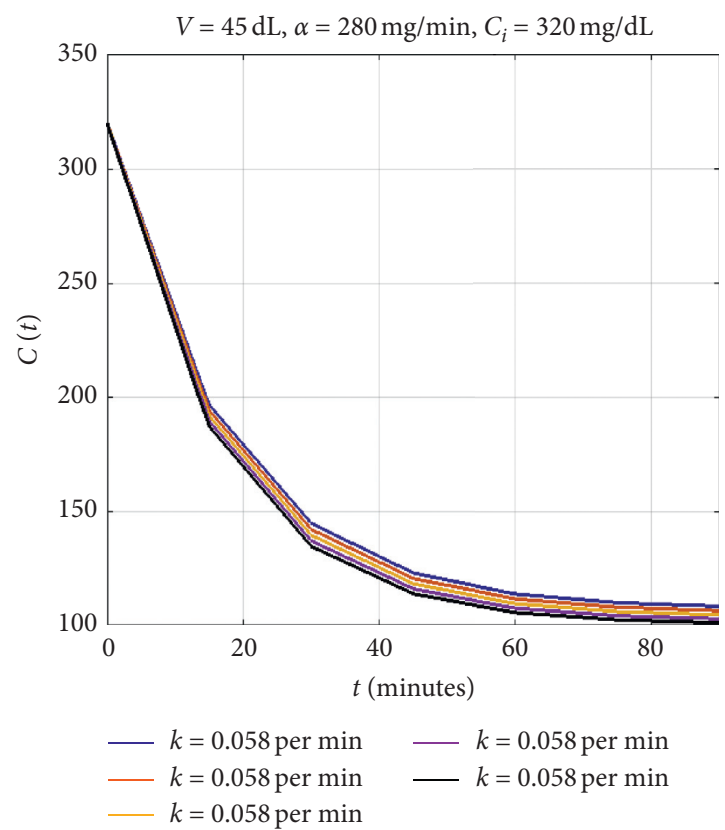

Figure 4: Blood glucose concentration $C(t)$ for different values of constant velocity of elimination $k$ and time $t$ with $V=45 \mathrm{dL}, a=280 \mathrm{mg} / \mathrm{min}$, and $C_{i}=320 \mathrm{mg} / \mathrm{dL}$.

methodology explained by taking numerical examples with application in the field of medical science during an intravenous injection (continuous) for determining blood glucose concentration of a patient at any particular time. Results of numerical examples depict that the Sawi decomposition method is a very fast and effective 
decomposition method for determining the solution of V.I.E. Furthermore, the Sawi decomposition method gives the solution of problem of blood glucose concentration and provides the information of required time to achieve normal blood glucose concentration, which is very useful for sugar patients and at the time of operation. The Sawi decomposition method will be useful for determining the primitives of system of V.I.E. and other problems of medical science, engineering, physical chemistry such as determination of tumor growth, counting the total number of infected cells, determining the concentration of viral particles in plasma during HIV-1 infections, examining the temperature effect on the vibration of skew plates, and determining the concentration of chemical substances of the chemical chain reaction in future.

\section{Data Availability}

The authors declare that the datasets used to support the finding of this paper are available from the corresponding author upon request.

\section{Conflicts of Interest}

The authors have no conflicts of interest regarding the publication of the paper.

\section{Acknowledgments}

This research was supported by Taif University Researchers Supporting Project Number (TURSP-2020/031), Taif University, Taif, Saudi Arabia.

\section{References}

[1] S. Aggarwal, R. Chauhan, and N. Sharma, "A new application of Kamal transform for solving linear Volterra integral equations," International Journal of Latest Technology in Engineering, Management \& Applied Science, vol. 7, no. 4, pp. 138-140, 2018.

[2] R. Chauhan and S. Aggarwal, "Laplace transform for convolution type linear Volterra integral equation of second kind," Journal of Advanced Research in Applied Mathematics and Statistics, vol. 4, no. 3\&4, pp. 1-7, 2019.

[3] M. M. M. Abdelrahim, "The new integral transform: Sawi transform," Advances in Theoretical and Applied Mathematics, vol. 14, no. 1, pp. 81-87, 2019.

[4] G. P. Singh and S. Aggarwal, "Sawi transform for population growth and decay problems," International Journal of Latest Technology in Engineering, Management \& Applied Science, vol. 8, no. 8, pp. 157-162, 2019.

[5] S. Aggarwal and A. R. Gupta, "Dualities between some useful integral transforms and Sawi transform," International Journal of Recent Technology and Engineering, vol. 8, no. 3, pp. 5978-5982, 2019.

[6] W. Wang, "A mechanical algorithm for solving the Volterra integral equation," Applied Mathematics and Computation, vol. 172, no. 2, pp. 1323-1341, 2006.

[7] K. Maleknejad and N. Aghazadeh, "Numerical solution of Volterra integral equations of the second kind with convolution kernel by using Taylor-series expansion method,"
Applied Mathematics and Computation, vol. 161, no. 3, pp. 915-922, 2005.

[8] J. Rashidinia and M. Zarebnia, "Solution of a Volterra integral equation by the sinc-collocation method," Journal of Computational and Applied Mathematics, vol. 206, no. 2, pp. 801-813, 2007.

[9] E. Babolian and A. Davari, "Numerical implementation of Adomian decomposition method for linear Volterra integral equations of the second kind," Applied Mathematics and Computation, vol. 165, no. 1, pp. 223-227, 2005.

[10] Q. Lin, I. H. Sloan, and R. Xie, "Extrapolation of the iteratedcollocation method for integral equations of the second kind," SIAM Journal on Numerical Analysis, vol. 27, no. 6, pp. 1535-1541, 1990.

[11] S. Zhang, Y. Lin, and M. Rao, "Numerical Solutions for second-kind Volterra integral equations by Galerkin methods," Applications of Mathematics, vol. 45, no. 1, pp. 19-39, 2000.

[12] S. E. Shoukralla and B. Magdy, "Barycentric - Maclaurin interpolation method for solving Volterra integral equations of the second kind," Menoufia Journal of Electronic Engineering Research, vol. 29, no. 1, pp. 84-90, 2020.

[13] S. A. Isaacson and R. M. Kirby, "Numerical solution of linear Volterra integral equations of the second kind with sharp gradients," Journal of Computational and Applied Mathematics, vol. 235, no. 14, pp. 4283-4301, 2011.

[14] A. E. Abaoud, A. S. Shkheam, and S. M. Zali, "The Adomian decomposition method of Volterra integral equation of second kind," American Journal of Applied Mathematics, vol. 6, no. 4, pp. 142-148, 2018.

[15] S. Aggarwal, R. Chauhan, and N. Sharma, "A new application of Mahgoub transform for solving linear Volterra integral equations," Asian Resonance, vol. 7, no. 2, pp. 46-48, 2018.

[16] S. Aggarwal, A. R. Gupta, and S. D. Sharma, "A new application of Shehu transform for handling Volterra integral equations of first kind," International Journal of Research in Advent Technology, vol. 7, no. 4, pp. 439-445, 2019.

[17] S. Aggarwal, N. Sharma, and R. Chauhan, "Solution of linear Volterra integral equations of second kind using Mohand transform," International Journal of Research in Advent Technology, vol. 6, no. 11, pp. 3098-3102, 2018.

[18] S. Aggarwal, N. Sharma, and R. Chauhan, "A new application of Aboodh transform for solving linear Volterra integral equations," Asian Resonance, vol. 7, no. 3, pp. 156-158, 2018.

[19] S. Aggarwal, N. Sharma, and R. Chauhan, "Duality relations of Kamal transform with Laplace, Laplace-Carson, Aboodh, Sumudu, Elzaki, Mohand and Sawi transforms," SN Applied Sciences, vol. 2, no. 1, p. 135, 2020.

[20] S. Aggarwal and K. Bhatnagar, "Dualities between Laplace transform and some useful integral transforms," International Journal of Engineering and Advanced Technology, vol. 9, no. 1, pp. 936-941, 2019.

[21] R. Chauhan, N. Kumar, and S. Aggarwal, "Dualities between Laplace-Carson transform and some useful integral transforms," International Journal of Innovative Technology and Exploring Engineering, vol. 8, no. 12, pp. 1654-1659, 2019.

[22] S. Aggarwal and A. R. Gupta, "Dualities between Mohand transform and some useful integral transforms," International Journal of Recent Technology and Engineering, vol. 8, no. 3, pp. 843-847, 2019.

[23] S. Aggarwal, K. Bhatnagar, and A. Dua, "Dualities between Elzaki transform and some useful integral transforms," International Journal of Innovative Technology and Exploring Engineering, vol. 8, no. 12, pp. 4312-4318, 2019. 
[24] R. Chaudhary, S. D. Sharma, N. Kumar, and S. Aggarwal, "Connections between Aboodh transform and some useful integral transforms," International Journal of Innovative Technology and Exploring Engineering, vol. 9, no. 1, pp. 1465-1470, 2019.

[25] S. Aggarwal, N. Sharma, and R. Chauhan, "Application of Mahgoub transform for solving linear Volterra integral equations of first kind," Global Journal of Engineering Science and Researches, vol. 5, no. 9, pp. 154-161, 2018.

[26] S. Aggarwal, R. Chauhan, and N. Sharma, "Application of Elzaki transform for solving linear Volterra integral equations of first kind," International Journal of Research in Advent Technology, vol. 6, no. 12, pp. 3687-3692, 2018.

[27] S. Aggarwal and N. Sharma, "Laplace transform for the solution of first kind linear Volterra integral equation," Journal of Advanced Research in Applied Mathematics and Statistics, vol. 4, no. 3\&4, pp. 16-23, 2019.

[28] R. Mishra, S. Aggarwal, L. Chaudhary, and A. Kumar, "Relationship between Sumudu and some efficient integral transforms," International Journal of Innovative Technology and Exploring Engineering, vol. 9, no. 3, pp. 153-159, 2020.

[29] P. Agarwal, A. Hyder, M. Zakarya, G. AlNemer, C. Cesarano, and D. Assante, "Exact solutions for a class of wick-type stochastic $(3+1)$-dimensional modified benjamin-bonamahony equations," Axioms, vol. 8, pp. 1-15, 2019.

[30] C. Cesarano, "Generalized special functions in the description of fractional diffusive equations," Communications in Applied and Industrial Mathematics, vol. 10, no. 1, pp. 31-40, 2019.

[31] G. Dattoli, P. E. Ricci, C. Cesarano, and L. Vázquez, "Special polynomials and fractional calculus," Mathematical and Computer Modelling, vol. 37, no. 7-8, pp. 729-733, 2003.

[32] S. Aggarwal, N. Sharma, and R. Chauhan, "Application of Aboodh transform for solving linear Volterra integral equations of first kind," International Journal of Research in Advent Technology, vol. 6, no. 12, pp. 3745-3753, 2018.

[33] S. Aggarwal, N. Sharma, and R. Chauhan, "Application of Kamal transform for solving linear Volterra integral equations of first kind," International Journal of Research in Advent Technology, vol. 6, no. 8, pp. 2081-2088, 2018.

[34] A. M. Wazwaz, A First Course in Integral Equations, World Scientific, Singapore, Singapore, 1997.

[35] B. L. Moiseiwitsch, Integral Equations, Longman, New York, NY, USA, 1977.

[36] A. M. Wazwaz, Linear and Nonlinear Integral Equations: Methods and Applications, Springer, London, UK, 2000.

[37] American Diabetes Association, "Screening for type 2 diabetes," Clinical Diabetes, vol. 18, no. 2, 2000. 